\title{
GSK-126 Protects CA1 Neurons from H3K27me3-Mediated Apoptosis in Cerebral Ischemia
}

\author{
Zhongcheng Wang ${ }^{1,2} \mathbb{D} \cdot$ Yaxin Su$^{1} \cdot$ Lei Zhang $^{2} \cdot \operatorname{Ting~}^{\mathrm{Lan}^{3}} \cdot \mathrm{Li} \mathrm{Li}^{1} \cdot$ Suhua $\mathrm{Qi}^{3}$
}

Received: 26 August 2021 / Accepted: 30 November 2021 / Published online: 29 January 2022

(c) The Author(s) 2021

\begin{abstract}
Epigenetics, including histone modifications, play a significant role in central nervous system diseases, but the underlying mechanism remains to be elucidated. The aim of this study was to evaluate the role of $\mathrm{H} 3 \mathrm{~K} 27 \mathrm{me} 3$ in regulating transcriptomic and pathogenic mechanisms following global ischemic stroke. Here, we found that in vivo ischemic/reperfusion (I/R) injury induced marked upregulation of H3K27me3 in the hippocampus. The administration of GSK-126 to rat brains decreased the levels of H3K27me3 in the hippocampus and reduced neuronal apoptosis after experimental stroke. Furthermore, ChIP-seq data demonstrated that the primary role of GSK-126 in the ischemic brain is to reduce H3K27me 3 enrichment, mediating negative regulation of the execution phase of apoptosis and the MAPK signaling pathway. Further study suggested that the protective role of GSK-126 in ischemic rats was antagonized by U0126, an inhibitor of ERK1/2. Collectively, we demonstrated the potential of $\mathrm{H} 3 \mathrm{~K} 27 \mathrm{me} 3$ as a novel stroke therapeutic target, and GSK-126 exerted a neuroprotective function in ischemic brain injury, which might be associated with activation of the MAPK/ERK pathway.
\end{abstract}

Keywords H3K27me3 · GSK-126 · Apoptosis · Neuroprotection · Global ischemia stroke

\section{Introduction}

Stroke is one of the leading causes of death and disability in the world, approximately $87 \%$ of which is ischemic stroke [1]. Both ischemic and hemorrhagic stroke lead to neuronal death. In response to global cerebral ischemia, hippocampal pyramidal neurons in the cornu ammonis 1 (CA1) region are particularly vulnerable, and apoptosis in the hippocampal CA1 region is the major cause of cell death [2]. Recognized

Zhongcheng Wang and Yaxin Su contributed equally to this work.

Zhongcheng Wang

wangzhongcheng@xzhmu.edu.cn

Suhua Qi

suhuaqi@xzhmu.edu.cn

1 Department of Pathophysiology, School of Basic Medical Sciences, Xuzhou Medical University, Xuzhou, China

2 Laboratory of Clinical and Experimental Pathology, School of Basic Medical Sciences, Xuzhou Medical University, Xuzhou, China

3 Department of Clinical Laboratory Diagnostics, School of Medical Technology, Xuzhou Medical University, Xuzhou, China mechanisms of neuronal death in stroke include excitotoxicity, calcium overload, and oxidative stress, but whether histone methylation, especially $\mathrm{H} 3 \mathrm{~K} 27 \mathrm{me} 3$, participates in these processes is still uncertain.

Emerging roles suggest that abnormal $\mathrm{H} 3 \mathrm{~K} 27 \mathrm{me} 3$ is found in many central nervous system diseases [3]. $\mathrm{H} 3 \mathrm{~K} 27 \mathrm{me} 3$ is an epigenetic gene repressor that plays a critical role in many neuronal processes, including neurodegeneration, synaptic function, epilepsy, and animal behavior [4-6]. Stroke is a disease of aging, and H3K27me3 levels increase with age $[7,8]$. Investigation into the relationship between H3K27me3 and stroke has increased. H3K27me3 is mediated by enhancer of zeste homolog-2 (EZH2), a histone methyltransferase of the catalytic component of polycomb repressor complex-2 (PCR2). Currently, few studies on $\mathrm{EZH} 2 / \mathrm{H} 3 \mathrm{~K} 27 \mathrm{me} 3$ in the prognosis or progression of stroke have involved neurons. As previously reported, inhibition of EZH2 activity promotes the differentiation of human plasma stem cells into neurons by increasing peroxisome proliferator-activated receptor gamma (PPARy) expression, and transplantation of human plasma stem cells with EZH2 knockdown into the ischemic brain promotes functional recovery from ischemic brain injury [9]. Moreover, EZH2 may regulate the activation of microglia by activating 
phosphorylated signal transducer and activator of transcription 3 (STAT3), aggravating the inflammatory response after ischemic stroke. The EZH2 inhibitor 3-deazaneplanocin A (DZnep) exerts a neuroprotective effect after ischemic stroke [10]. Another EZH2 inhibitor, GSK-126, was also found to be safe for use in the central nervous system [11]. Therefore, in the present study, we investigated whether H3K27me3 was involved in the risk of stroke and whether GSK-126 may be used for the prevention and treatment of stroke.

In brief, the relationship between $\mathrm{H} 3 \mathrm{~K} 27 \mathrm{me} 3$ and neuronal death in the ischemic brain was characterized in the present study. In addition, this study also revealed that H3K27me3 may participate in the regulation of apoptosis through the MAPK pathway in the ischemic brain. These findings are not only beneficial for us to understand the role of $\mathrm{H} 3 \mathrm{~K} 27 \mathrm{me} 3$ in the pathogenesis of stroke but also suggest the potential value of GSK-126 in the prevention and treatment of stroke.

\section{Materials and Methods}

\section{Animals and Ethics}

Sprague-Dawley rats (male) weighing 230-250 g were obtained from the laboratory animal center of Xuzhou Medical University. The animals were housed in groups of 4-5 rats/cage with free access to food and water under a 12 light/12 dark cycle. In the present study, rats were randomly assigned to the experimental groups. All animal procedures were approved by the committee on the ethics of animal experiments of Xuzhou Medical University (D2016017), and animals were cared for in accordance with the Guide for the Care and Use of Laboratory Animals.

\section{Preparation of the Transient Global Cerebral Ischemia Model}

We utilized a model of global cerebral ischemia and reperfusion induced by four vessel occlusions as previously described [12, 13]. In brief, rats were anesthetized with isoflurane, both vertebral arteries were permanently electrocauterized, and the common carotid arteries were isolated. After $24 \mathrm{~h}$, the common carotid arteries were re-exposed and clipped using artery clips for $15 \mathrm{~min}$ followed by reperfusion. During the entire procedure, a heating pad and heating lamps were used to maintain the rectal temperature at 37 ${ }^{\circ} \mathrm{C}$. Rats that lost their righting reflex within $60 \mathrm{~s}$, those that lost consciousness, and those that exhibited white eyeballs during ischemia were selected for subsequent experiments. Sham-operated control rats were subjected to the same procedure except for occlusion of the carotid artery. After $24 \mathrm{~h}$ of ischemia and reperfusion, modified neurological severity score (mNSS) was adopted to evaluate the neurological function of rats in each group [14]. In this study, the animal survival rate was approximately $65-75 \%$, and the model success rate was $75 \%$.

\section{Drugs and Administration}

For the surgery, rats were anesthetized using isoflurane and placed into a stereotactic device with lambda and bregma at a horizontal level. A midline incision of the skin was made to unbar the skull, and a small hole was drilled into the skull using the bregma as a reference (stereotaxic zero) at the following stereotactic coordinates: mediolateral (ML): $1.0 \mathrm{~mm}$ away from midline on the left side; anteroposterior (AP): $1.5 \mathrm{~mm}$ posterior to the bregma, which corresponded to the lateral ventricle region, based on the anatomical atlas of the rat brain. The catheter was inserted into the small hole and secured using dental cement. To eliminate inflammation and repair the blood-brain barrier, the rats were placed back into their cages and allowed to rest for 3 days. For the intracerebroventricular injection (i.c.v.), rats were anesthetized using isoflurane, and drugs or normal saline were injected starting on the fourth day.

GSK-126 and U0126 were obtained from Vicmed Biotech (Xuzhou, China). The powder formulations were stored at $-20{ }^{\circ} \mathrm{C}$ and dissolved in $20 \%$ Captisol adjusted to $\mathrm{pH} 4-4.5$ using $1 \mathrm{~N}$ acetic acid before use in in vivo studies at a final dissolved concentration of $5 \mathrm{mg} / \mathrm{ml}$. GSK-126 was injected into the lateral ventricle for 7 consecutive days ( $20 \mu \mathrm{l} /$ day, i.c.v.) before global cerebral ischemia modeling, and U0126 was administered $30 \mathrm{~min}$ prior to ischemia $(5 \mu \mathrm{l}, 0.2 \mu \mathrm{g} /$ $\mu 1$, i.c.v.). Rats in the sham group were administered equal volumes of normal saline. All drugs were injected into the lateral ventricle by using a mini pump (R462, RWD Biotech, Shenzhen, China).

\section{Western Blot}

Rat hippocampal tissues were collected $24 \mathrm{~h}$ after reperfusion. Protein samples (40-50 $\mu \mathrm{g}$ in each lane) were loaded onto SDS PAGE gels for electrophoresis. Proteins were transferred to polyvinylidene difluoride membranes and then blocked in 5\% dried milk at room temperature. Primary antibodies were added and incubated at $4{ }^{\circ} \mathrm{C}$ overnight. H3K27me3 (mAbcam6002) and histone H3 (mAbcam 10799) were purchased from Abcam (Shanghai, China), and $\beta$-actin (ab179467) and caspase-3 (196771-AP) were purchased from Proteintech (Wuhan, China). Then, the membrane was incubated with the secondary antibody (dilution 1:5000) at $37^{\circ} \mathrm{C}$ for $2 \mathrm{~h}$. The protein bands were detected using a chemiluminescent substrate kit (Merck Millipore, USA) according to the manufacturer's 
recommendations. Band intensities were quantified using Quantity One software.

\section{Nissl Staining}

After 15 min of ischemia and 5 days of reperfusion, the rats were given isoflurane anesthesia and euthanized to remove the intact brain tissue, which was placed in $4 \%$ paraformaldehyde and fixed for $12 \mathrm{~h}$. Coronal sections (10- $\mu \mathrm{m}$ thick) were cut and stored at $-80{ }^{\circ} \mathrm{C}$. Every hippocampus section was selected for Nissl staining using $0.3 \%$ cresyl violet to evaluate infarct volumes.

\section{TUNEL Staining}

After $15 \mathrm{~min}$ of ischemia and $24 \mathrm{~h}$ of reperfusion, the brain tissues were removed as it was mentioned above. In brief, sections were dewaxed by conventional methods, and then were incubated with $1 \%$ Triton- 100 for $15 \mathrm{~min}$ at room temperature, and subsequently incubated with $3 \% \mathrm{H}_{2} \mathrm{O}_{2}$ for 15 min at room temperature. Finally, TUNEL reaction mixture was then added to each sample and then incubated at $37^{\circ} \mathrm{C}$ for $60 \mathrm{~min}$.

\section{Chromatin Immunoprecipitation Sequencing (ChIP-seq) and Data Mining}

Hippocampal tissues were collected after $24 \mathrm{~h}$ of reperfusion. ChIP analysis was performed using a chromatin immunoprecipitation kit (Millipore, USA) according to the manufacturer's instructions. Immunoprecipitated and input DNA was purified and transferred to CloudSeq Biotech (Shanghai, China) to perform high-throughput sequencing. Raw data were generated after sequencing, and image analysis, base calling, and quality filtering were performed on an Illumina NovaSeq 6000 sequencer. Peak calling was performed using MACS software. Differentially enriched regions $(p<0.05)$ were identified using diffReps software. The enriched peaks were then annotated using the latest UCSC RefSeq database to link the peak information to the gene annotation.

Gene ontology (GO) and pathway analyses were performed on the peak-associated genes or differentially enriched peak-associated genes. GO analysis was performed to elucidate the biological implications of unique genes in the significance experiment. The "elim Fisher" algorithm was used for the GO enrichment test. GO categories with a $p<0.01$ are reported. The gene ontology is structured as a directed acyclic graph, and each term has defined relationships to one or more other terms. Pathway analysis was implemented to determine the significant pathways of the peak-associated genes according to the KEGG database. Fisher's exact test was used to identify significant enrichment for pathways; pathway categories with $p<0.01$ are reported.

\section{Statistics}

All data were analyzed by an investigator blinded to the group allocation. GraphPad Prism 5 was used for all data analysis. Throughout the text, summary data are presented as the means \pm SEM. One-way ANOVA and $T$-tests were used to assess the statistical significance of the differences between groups.

\section{Results}

\section{GSK-126 Decreases H3K27me3 Levels in the Rat Hippocampus}

We speculated that during the ischemia reperfusion process, levels of H3K27me3 increased in the brain. To test this hypothesis, we initially examined whether ischemia increases $\mathrm{H} 3 \mathrm{~K} 27 \mathrm{me} 3$ in the hippocampus. A remarkable increase in the levels of $\mathrm{H} 3 \mathrm{~K} 27 \mathrm{me} 3$ was observed in the $\mathrm{I} / \mathrm{R}$ groups, and this increase in $\mathrm{H} 3 \mathrm{~K} 27 \mathrm{me} 3$ was not due to abnormal EZH2 expression (Fig. 1A-C). To determine whether GSK-126 decreased the levels of H3K27me3 in the hippocampus, we next performed a time effect test. As shown in Fig. 2A-C, levels of H3K27me3 in the hippocampus were successfully reduced by i.c.v. injection of GSK-126 for 7 consecutive days. Therefore, the global cerebral ischemia model surgery was performed 7 days after injection of GSK-126 (Fig. 2D). Moreover, compared to the I/R group, rats preadministered GSK-126 exhibited reduced $\mathrm{H} 3 \mathrm{~K} 27 \mathrm{me} 3$ levels in response to ischemia induction (Fig. 2E-F).

\section{GSK-126 Protects CA1 Neuron Survival in Response to Ischemia}

To determine the effect of GSK-126 on brain ischemia and reperfusion, Nissl staining was performed to detect changes in the number of hippocampal neurons. As shown in Fig. 3A-B, pyramidal neurons in the CA1 region were particularly vulnerable to ischemia, but GSK-126 protected CA1 neurons from ischemia. GSK-126 itself did not affect the arrangement, shape, or conformation of pyramidal cells in the CA1 region. As apoptosis in the hippocampal CA1 region is the major cause of cell death, we next examined whether GSK-126 reduced CA1 neurons apoptosis. A significant increase in the level of cleaved caspase- 3 , but not caspase-3, was observed in ischemic rats, and which could be reversed by GSK-126 (Fig. 3C-D). Combined with the TUNEL staining (Fig. S1), we could conclude that protective 


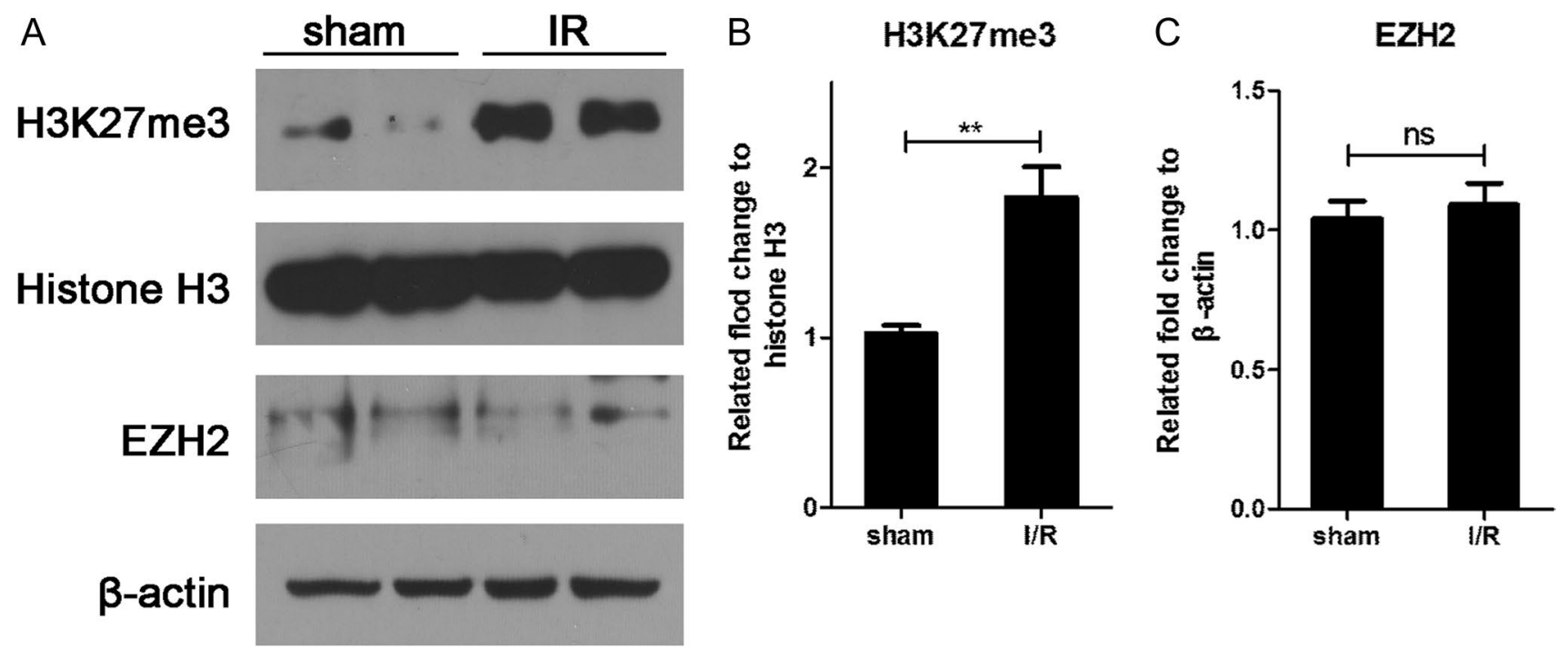

Fig. 1 H3K27me3 levels are elevated in the ischemic rat brain. A Representative levels of H3K27me3 and EZH2 in I/R rats. Histone $\mathrm{H} 3$ and $\beta$-actin were used as loading controls. B The relative inten-

role of GSK-126 on CA1 neurons after I/R was due to its inhibitory effect on apoptosis. In addition, according to the mNSS score, GSK-126 can improve the behavioral performance in ischemic rats (Fig. 3E). In brief, this result demonstrated that GSK-126 has potential as a treatment for stroke.

\section{GSK-126 May Inhibit Apoptosis via the MAPK Pathway in the Ischemic Brain}

To further determine the mechanisms of GSK-126 in protecting neuronal survival in response to ischemia, ChIP-seq was performed to determine the variation in $\mathrm{H} 3 \mathrm{~K} 27 \mathrm{me} 3$ enrichment in the whole genome (Fig. 4A). As the role of GSK-126 was to inhibit levels of H3K27me3, we were primarily concerned about the differences between ischemic rats preadministered GSK-126 and control ischemic rats. Due to the extensive inhibitory effects of H3K27me3 on gene expression, increased $\mathrm{H} 3 \mathrm{~K} 27 \mathrm{me} 3$ was found to be enriched in 12050 gene sequences in ischemic rats compared to sham rats, while decreased H3K27me3 levels were found to be enriched in 13449 gene sequences in ischemic rats pretreated with GSK-126 compared to sham rats. Furthermore, decreased H3K27me3 enrichment was found on 12108 genes in ischemic rats pretreated with GSK-126 compared to ischemic rats. However, these three groups shared 9112 genes in common, suggesting that the increased enrichment of $\mathrm{H} 3 \mathrm{~K} 27 \mathrm{me} 3$ on most genes caused by ischemia could be reversed by GSK-126 (Fig. 4B). We speculate that genes upregulated in ischemic rats preadministered GSK-126 are responsible for the neuroprotective effects of GSK-126. Therefore, H3K27me3 should be less sity of H3K27me3 illustrated in panel A. C The relative intensity of EZH2 illustrated in panel A. ${ }^{* *} p<0.01$

enriched on those gene sequences. The GO category results confirmed this hypothesis. In contrast to ischemic rats, the most significantly different GO terms for genes with reduced H3K27me3 enrichment in I/R rats pretreated with GSK-126 compared to I/R rats were associated with negative regulation of apoptosis (Fig. 4C, Table S1). In addition, pathway analysis of the above genes revealed a remarkable decrease in $\mathrm{H} 3 \mathrm{~K} 27 \mathrm{me} 3$ enrichment in genes involved in the MAPK pathway (Fig. 4D, Fig. S2A, Table S2). MAPKs mediate intracellular signaling pathways associated with cell death and survival in stroke, which has been demonstrated by numerous studies $[15,16]$. Whether GSK-126 protects neurons from apoptosis by activating the MAPK pathway is a hypothesis worth exploring.

\section{U0126 Antagonizes the Antiapoptotic Effect of GSK-126 in the Ischemia/Reperfusion Brain}

Among the MAPK pathway genes mentioned above, several key genes of the MAPK/ERK1 signaling pathway were involved (Table S2). The extracellular signalregulated kinase $1 / 2$ (ERK1/2) signaling cascade plays a vital role in promoting neuronal survival during focal and global brain ischemic injury, and U0126 is a specific inhibitor of ERK1/2 [17-19]. To investigate whether GSK-126 protects neurons from apoptosis by activating the ERK1 pathway, we first examined whether GSK-126 treatment modulating ERK activity. As it was shown in Fig. 4E, significant increase in the levels of p-ERK and ERK was observed in ischemic rats that were treated with GSK-126. The interesting thing is that the increasing 


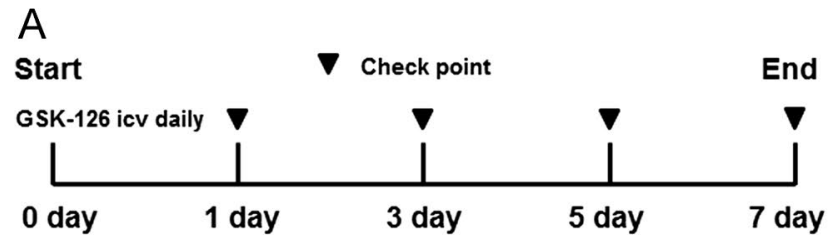

B

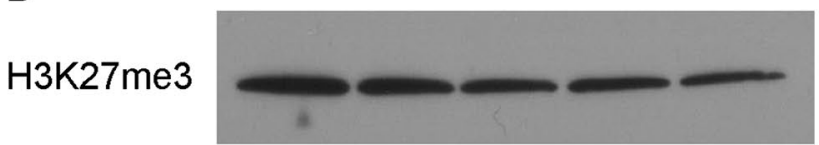

Histone $\mathrm{H} 3$

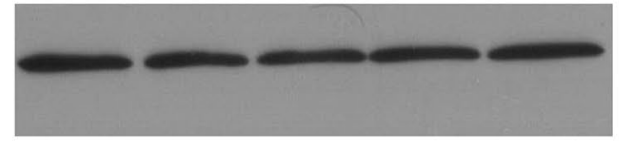

Day

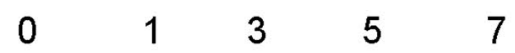

C

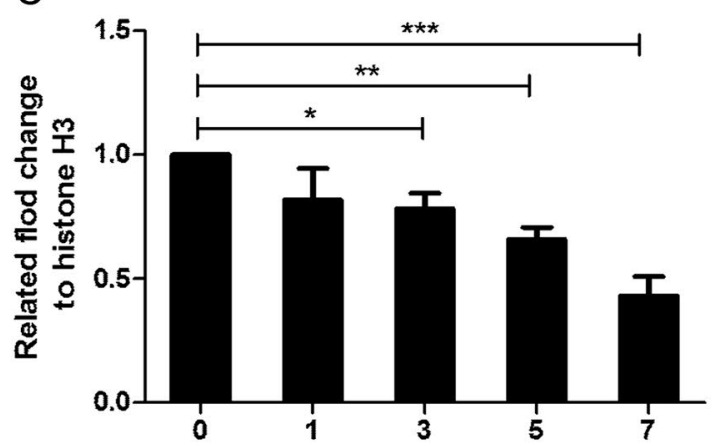

Fig. 2 GSK-126 reduces levels of H3K27me3 in the rat hippocampus in response to ischemia. A Schematic diagram of the time course experiment. B Protein extracts from the rat hippocampus were probed by western blotting with $\mathrm{H} 3 \mathrm{~K} 27 \mathrm{me} 3$ antibodies. Histone $\mathrm{H} 3$ was used as a loading control. Protein levels of H3K27me3 decreased after 7 consecutive days of injection, and GSK-126 was injected for 7 consecutive days in subsequent experiments. C Relative intensity of H3K27me3 illustrated in panel B. D Schematic diagram of GSK-126

level of p-ERK was highly associated with the expression level of ERK (Fig. 4E, Fig. S3). To provide the evidence to verify that ERK pathway was critical to GSK-126 to protect CA1 neurons after I/R, U0126 was administered to rats pretreated with GSK-126 before ischemia. The protective role of GSK-126 in ischemic rats was antagonized by U0126 (Fig. 5, Fig. S1, and Fig. S4). Consistent with the Nissl's staining result, a significant increase in cleaved caspase- 3 was observed in ischemic rats, which was reversed by pretreatment GSK-126. However, the reduction in cleaved caspase- 3 caused by GSK-126 after ischemia was antagonized by the administration of U0126. Moreover, changes in the levels of cleaved caspase- 3 were independent of the expression of caspase-3 (Fig. 5C-D). In

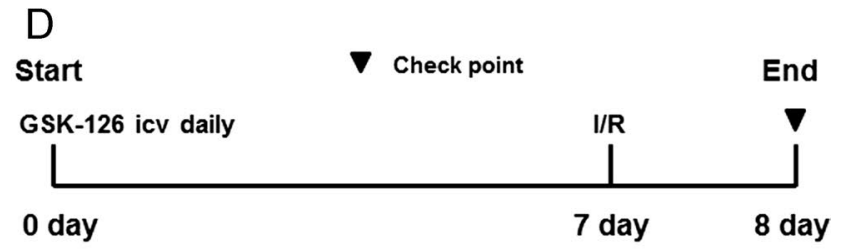

E H3K27me3
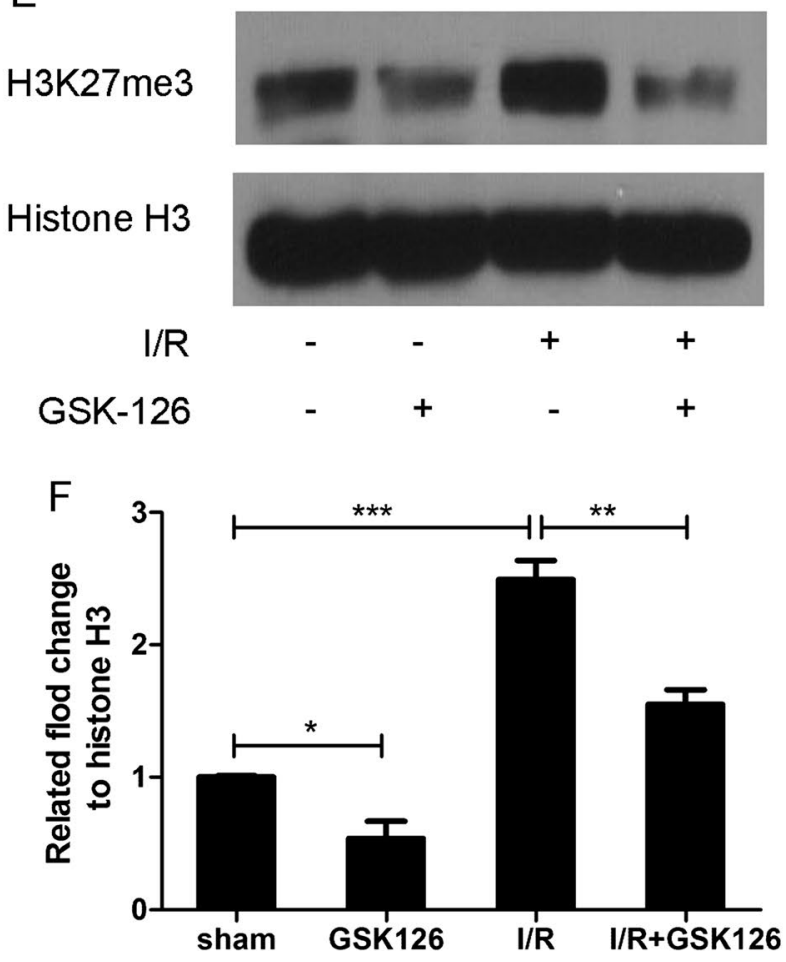

administration and global brain ischemia modeling. Global cerebral ischemia model surgery was performed after rats were administered GSK-126 for 7 consecutive days. E Compared to I/R rats, I/R rats preadministered GSK-126 exhibited reduced H3K27me3 levels in the hippocampus. Protein extracts from the rat hippocampus were probed on western blots with $\mathrm{H} 3 \mathrm{~K} 27 \mathrm{me} 3$ antibodies. Histone $\mathrm{H} 3$ was used as a loading control. F Relative intensity of H3K27me3 illustrated in panel E. $* p<0.05$. $* * p<0.01 . * * * p<0.001 . N=3$

brief, U0126 antagonizes the antiapoptotic effect of GSK126 in animal models of cerebral ischemia.

\section{Discussion}

Ischemic stroke is initiated by the interruption of cerebral blood flow and is followed by reperfusion, which further induces neuronal death, leading to brain tissue injury. Apoptosis is the primary mechanism of cell death and is regulated by several signaling pathways [20]. Investigation of novel treatment strategies to target cell death is still needed. Related studies have found that histone methylase inhibitors and histone demethylase inhibitors have effects in 
A
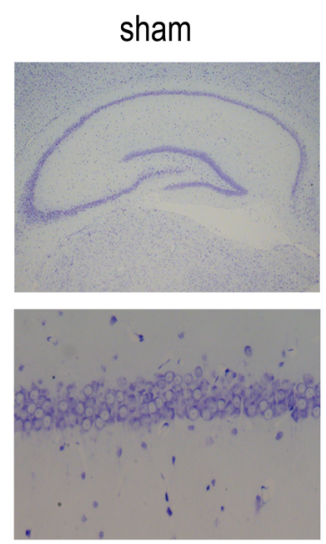

C

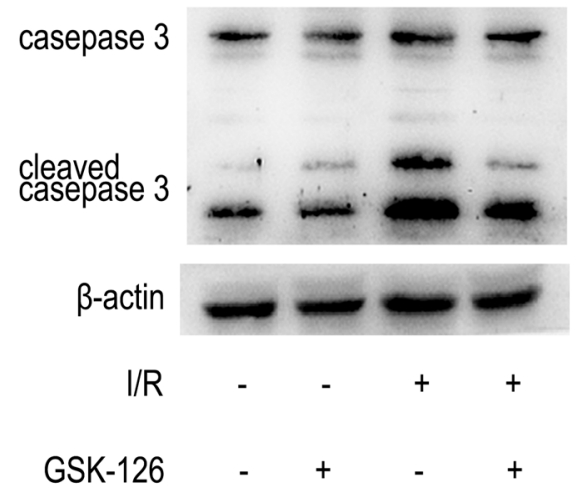

GSK-126
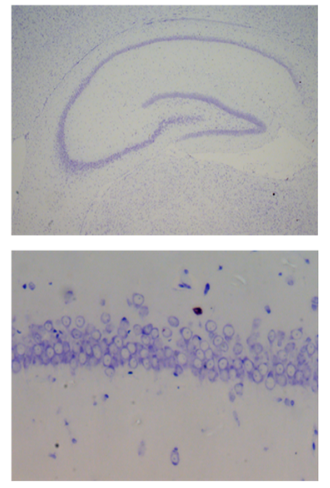

D

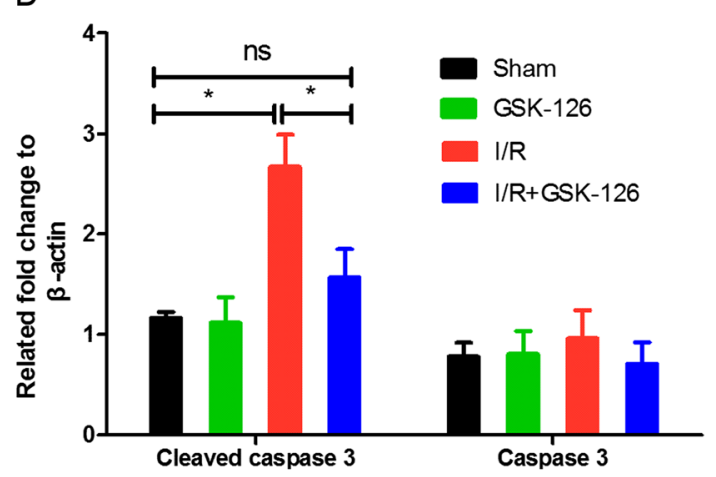

B

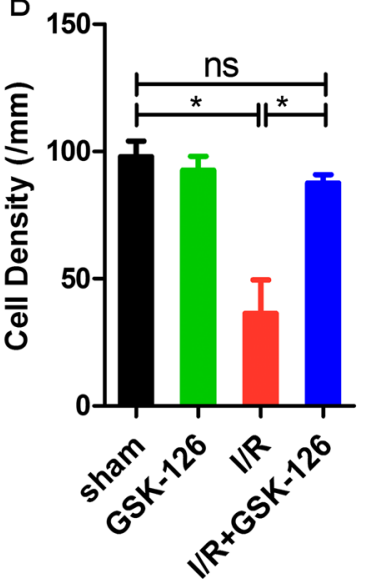

E

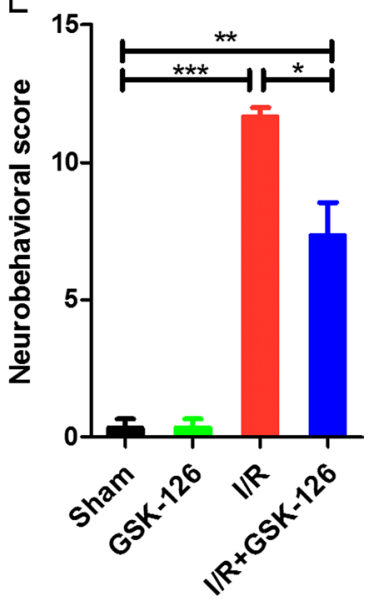

Fig. 3 GSK-126 decreases CA1 neuronal apoptosis after ischemia. A Nissl staining was performed on rat hippocampal sections. The boxed areas (CA1 areas) in the upper row are shown at higher magnification in the second row. The scale bar is $500 \mathrm{~mm}$ in the top line and $50 \mu \mathrm{m}$ in the bottom line. B Quantitative analysis of the above data, which were obtained from three rats in each group, and the results of a typical experiment are presented. C Representative levels of caspase-3

the treatment of ischemic stroke [21]. However, they have rarely been reported to be related to the apoptotic pathway of ischemic stroke. In this study, we demonstrated that the EZH2 inhibitor GSK-126 alleviated neuronal apoptosis induced by ischemic stroke. Moreover, MAPK/ERK pathway activation was required for this process (Fig. 6).

Methylation levels of H3K27 are mediated by the histone methylase EZH2 and the histone demethylase JMJD3. $\mathrm{H} 3 \mathrm{~K} 27 \mathrm{me} 3$ is involved in a variety of biological processes by inhibiting gene expression. However, the role of H3K27me3 in neuronal death has been less well investigated. A previous report revealed that JMJD3 rescues apoptosis in hippocampal neurons by upregulating the expression of BDNF, and JMJD3 plays an important role in the expression of Bax and caspase-3 during oxygen-glucose deprivation (OGD) injury $[22,23]$. In addition, we previously found that elevation of and cleaved caspase- 3 in each group. Protein extracts from the rat hippocampus were probed on western blots using caspase-3/cleaved caspase- 3 antibodies. $\beta$-actin was used as a loading control. D Relative intensity of caspase- 3 and cleaved caspase- 3 illustrated in panel C. $\mathbf{E}$ The behavioral evaluation by the mNSS test. $* p<0.05$, ** $p<$ $0.01, * * * p<0.001, \mathrm{~ns}$ indicates no significant differences. $N=6$

H3K27me3 downregulated the expression of $\mathrm{Na}^{+} / \mathrm{Ca}^{2+}$ exchanger 3 (NCX3) in hippocampal neurons, while GSK126 restored expression levels of NCX3 [11]. NCX3 is a gene that reduces neuronal death after ischemic brain damage [24]. Combined with our observation in I/R rats, these results indicate that the increasing $\mathrm{H} 3 \mathrm{~K} 27 \mathrm{me} 3$ levels in the brains of I/R rats were independent of the expression of EZH2 (Fig. 1). It would be interesting to examine whether an EZH2 inhibitor such as GSK-126 protects neurons from ischemic brain injury-induced neuron death.

GSK-126 was originally developed as a more effective inhibitor of EZH2 [25]. It exhibits higher selective inhibition of EZH2 than other EZH2 inhibitors, such as DZNep and EPZ005687 [26]. Moreover, GSK-126 has entered phase I clinical trials [27]. However, low oral bioavailability and blood-brain barrier permeability issues with GSK-126 

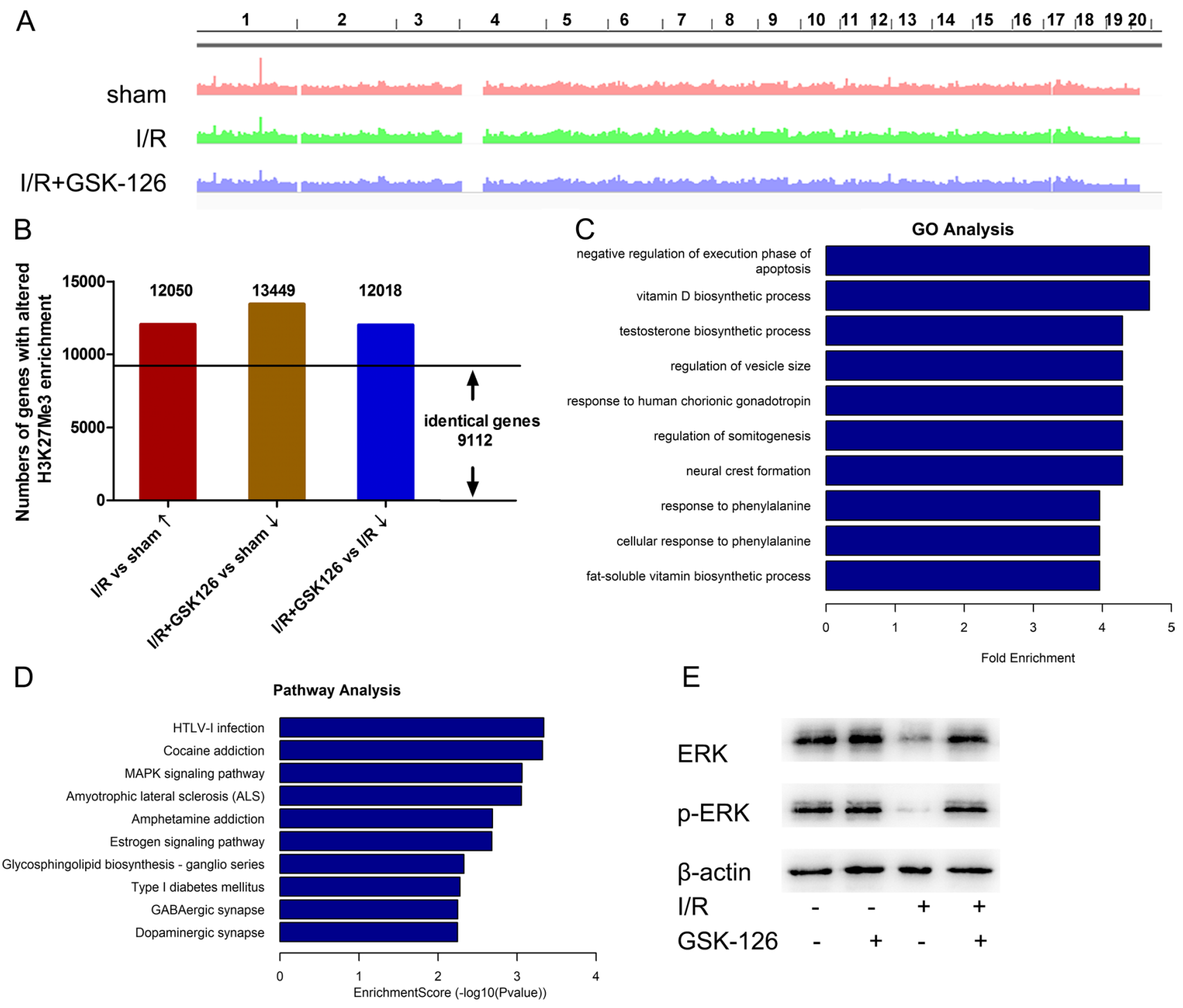

Fig. 4 ChIP-seq analysis. A An illustration of H3K27me3 enrichment in the entire genome. B Numbers of genes with differential enrichment of H3K27me3 in each group. The arrow after the group name indicates upregulated or downregulated enrichment levels of H3K27me3 in the specified group. C Significant GO category for genes with downregulated enrichment levels of H3K27me3 between I/R rats pretreated with GSK-126 and I/R rats. D Top 10 signifi-

were observed in animal models, which may limit its use in the central nervous system [28]. In contrast, reports have suggested that large doses of intraperitoneally administered GSK-126 reduce levels of H3K27me3 in the brain [11]. Therefore, to investigate the role of $\mathrm{H} 3 \mathrm{~K} 27 \mathrm{me} 3$ in the ischemic brain, GSK-126 was administered to rats by intracerebroventricular injection in the present study. A time course experiment was performed and revealed that levels of $\mathrm{H} 3 \mathrm{~K} 27 \mathrm{me} 3$ in the hippocampus were reduced in response to 7 consecutive days of intracerebroventricular injection of GSK-126 (Fig. 2A-C). Rats preadministered GSK-126 cant pathways for genes with downregulated enrichment levels of H3K27me3 between the I/R rats pretreated with GSK-126 and I/R rats. E Representative levels of ERK and p-ERK in each group. Protein extracts from the rat hippocampus were probed on western blots using ERK and p-ERK antibodies, respectively. $\beta$-actin was used as a loading control

exhibited attenuated H3K27me3 levels (Fig. 2D-F) and more surviving neurons in response to I/R (Fig. 3).

The mechanism by which GSK-126 affects neuronal death is still unknown. According to the ChIP-seq analysis, the essential genes bound and differentially enriched by H3K27me3 span a multitude of various genes (Fig. 4A). Because GSK-126 inhibited levels of H3K27me3, we primarily analyzed the difference in H3K27me3 enrichment between I/R rats preadministered GSK-126 and I/R rats, along with control animals. Interestingly, we found that the three groups shared 9112 identical genes (Fig. 4B). 

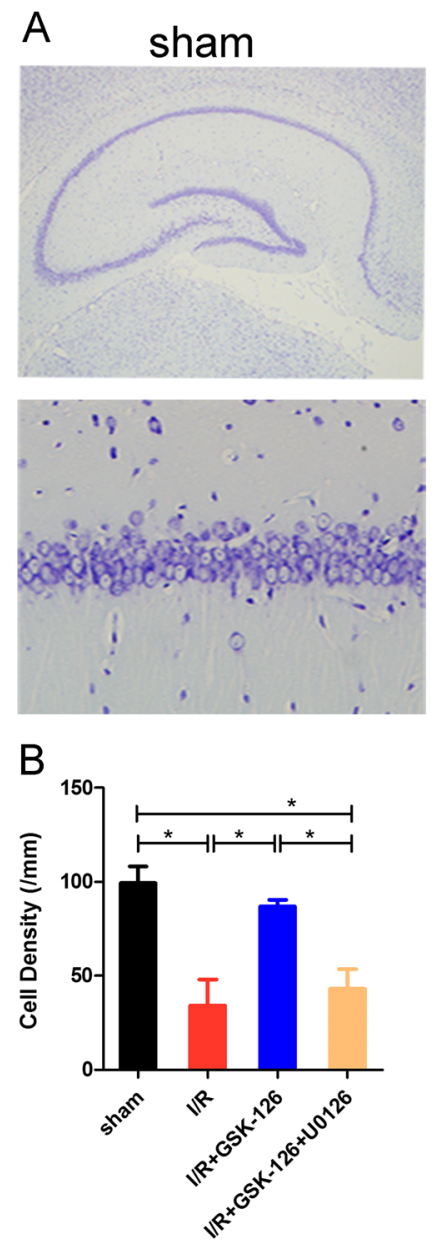
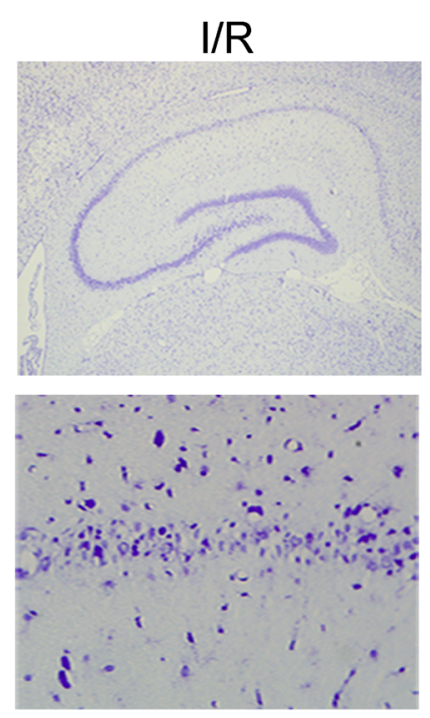

C

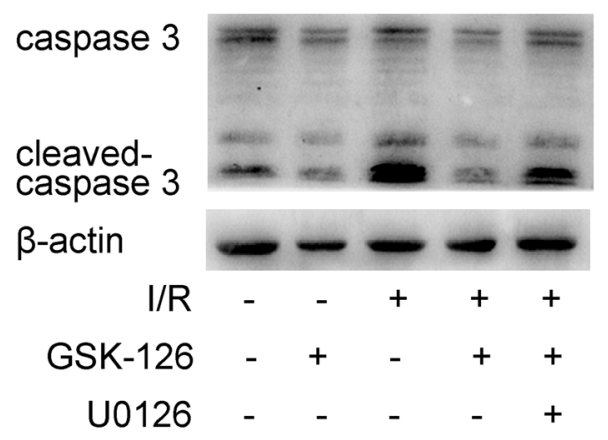

I/R+GSK-126
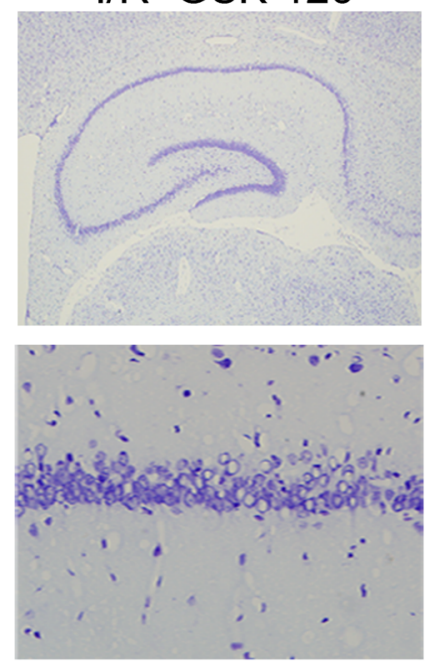

D
I/R+GSK-126+U0126
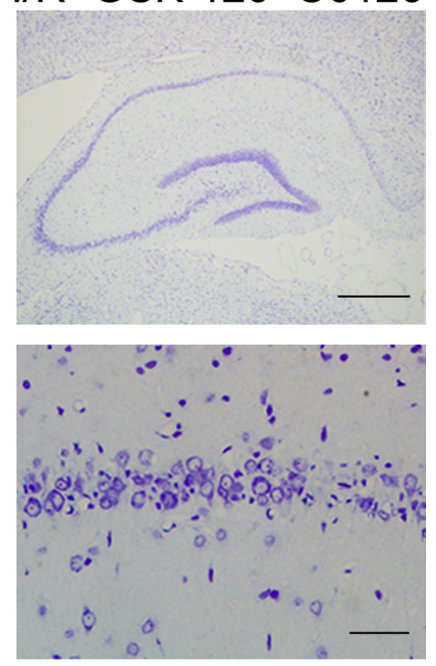

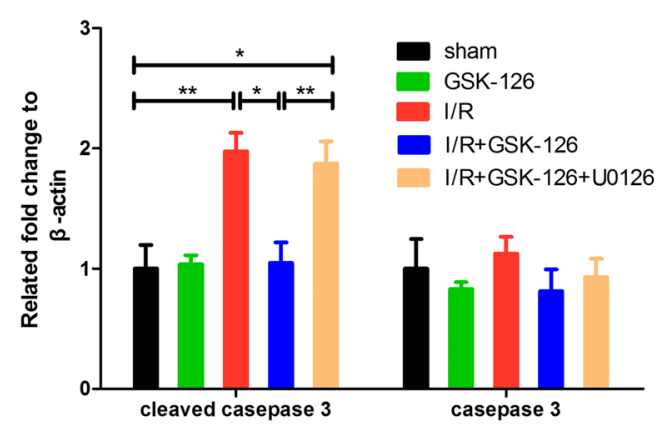

Fig. 5 U0126 antagonizes the protective effect of GSK-126 in the I/R brain. A Nissl staining was performed on rat hippocampal sections. The upper line shows neurons in the hippocampus of each group. The boxed areas (CA1 areas) in the upper line are higher magnification of the bottom line. Scale bar, $50 \mu \mathrm{m}$. B Quantitative analysis of the above data, which were obtained from three rats in each group, and the results of a typical experiment are presented. C Representative levels of caspase-3/cleaved caspase- 3 in each group. Protein extracts from the rat hippocampus were probed on western blots using caspase-3/cleaved caspase- 3 antibodies. $\beta$-actin was used as a loading control. D Relative intensity of caspase- 3 and cleaved caspase- 3 illustrated in panel C. $* p<0.05, * * p<0.01 . N=3$
These identical genes indicate that most genes affected by increasing levels of $\mathrm{H} 3 \mathrm{~K} 27 \mathrm{me} 3$ after ischemia were reversed by GSK-126. Therefore, GO analyses were performed to detect the potential relationship between these genes and the pathology of ischemic stroke. The results revealed that negative regulation of the execution phase of apoptosis was the most significantly altered GO, and compared to ischemic rats, ischemic rats preadministered GSK-126 negatively regulated antiapoptotic genes (DFFA, NMAT1, PAM16, BCL2L1, FZD3, and CXCR3) (Fig. 4C, Table S1). Among these genes, NMAT1 and BCL2L1 are responsible for reducing cell death and apoptosis after ischemic stroke [29, 30]. CXCR3, the receptor of CXCL10, is known to reduce brain infarction and attenuate BBB disruption in stroke [31]. In addition, the known causes of neuronal apoptosis after stroke include reactive oxygen species (ROS), calcium overload, excitatory toxicity, and inflammatory reactions. ROS are one of the early and most important components of cerebral brain ischemia, and excessive production of ROS leads to oxidative injury, including DNA damage, protein oxidation, and lipid peroxidation, ultimately leading to apoptosis [32]. After ischemic stroke, ATP deficiency leads to $\mathrm{Ca}^{2+}$ overload, which can induce neuronal apoptosis [33, 34]. Moreover, cerebral ischemia results in release of large amounts of glutamate, which stimulates NMDARs and induces calcium influx through these ionotropic receptors. The calciumdependent activation of death-signaling proteins that are immediately downstream of the receptors triggers excessive signaling cascades that work cooperatively to induce neuronal death [35]. Therefore, excitotoxicity is considered one of the most important mechanisms of neuronal apoptosis after ischemia [36]. In the present study, the GO results were consistent with the above research. Altered $G$ proteincoupled glutamate receptor signaling pathways were also 


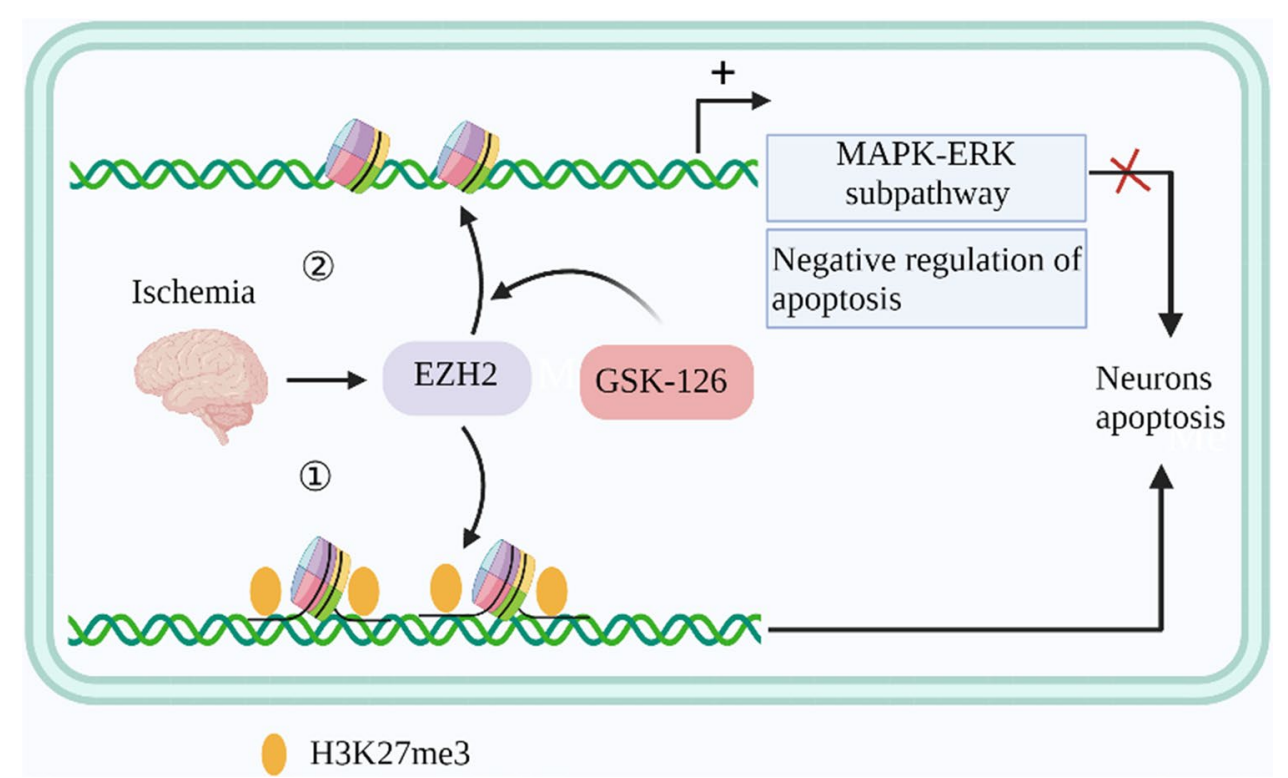

Fig. 6 A schematic illustration of the possible mechanism by which GSK-126 protects neurons from apoptosis. (1) After ischemic stroke, the levels of $\mathrm{H} 3 \mathrm{~K} 27 \mathrm{me} 3$ are increased by EZH2, leading to neuronal apoptosis. (2) GSK-126 reduces the expression levels of H3K27me3 by interfering with the activity of EZH 2 and reduces the enrichment

identified between sham rats and I/R rats pretreated with GSK-126 (Fig. S2B, Table S3).

The KEGG pathway analysis suggested that the MAPK signaling pathway was one of the most significantly altered pathways. The MAPK pathway consists of three subpathways: ERK-MAPK, JNK-MAPK, and p38-MAPK. ERK1/2, JNK, and p38 can induce both cell survival and cell death in response to ischemic stroke [20]. Among them, ERK1/2 was reported to play an antiapoptotic role in the process of ischemic brain injury, while JNK and $\mathrm{p} 38$ promote apoptosis after ischemia [37, 38]. Here, we found that 40 genes in the MAPK pathway were affected by H3K27me3 enrichment (Fig. 4D, Table S2). In addition, several key genes (BDNF, MEKK2, MYC, etc.) in the ERK1/2 pathway were included in these 40 genes. ERK can be activated by BDNF, MEK is an upstream protein of ERK, and MYC is activated by ERK $[20,39,40]$. This evidence suggests that GSK-126 may activate the entire ERK1/2 signaling pathway in the brains of $\mathrm{I} / \mathrm{R}$ rats.

To test the above hypothesis, rats were administered the MEK inhibitor U0126 after 7 days of GSK-126 injection to block the ERK1/2 pathway before ischemia modeling. As expected, the role of GSK-126 in protecting CA1 neurons from ischemia-induced apoptosis was antagonized by administration of U0126 (Fig. 5, Fig. S1). Levels of cleaved caspase- 3 further confirmed this conclusion. Our results indicate that activation of the ERK $1 / 2$ pathway may contribute to the reduction of neuronal apoptosis of $\mathrm{H} 3 \mathrm{~K} 27 \mathrm{me} 3$ on gene sequences, including genes related to negative regulation of apoptosis and the MAPK pathway. These genes are helpful for inhibiting neuronal apoptosis. Moreover, U0126 targets EK1/2 to antagonize the effects of GSK-126 in preserving neurons in the ischemic brain. The orange plot indicates H3K27me3

after ischemia. Nevertheless, whether ERK activation promotes or inhibits neuronal apoptosis is still uncertain. Some reports indicate that inhibition of the ERK signaling pathway by U0126 inhibits apoptosis after ischemia [41, 42]. These conflicting results may be due to the complexity of the MAPK signaling pathway and the inconsistency among experimental conditions. In our research, it would be helpful to further study the impact of H3K27me3 on the JNK-MAPK and p38-MAPK pathways.

In addition, the GABAergic synapse pathway was among the top 10 significantly altered pathways in $\mathrm{I} / \mathrm{R}$ rats preadministered GSK-126 compared to I/R rats (Fig. 4D), which is in line with the findings that GABA has a protective effect in ischemic stroke $[43,44]$. During an ischemic episode, the extracellular cerebral GABA concentration increases. Related studies have revealed that GABAergic signaling is enhanced through pharmacological intervention just hours after stroke; neuroprotection can be achieved by reducing the excitotoxic index and lowering the release of glutamate $[45,46]$. These reports further validate the accuracy of our ChIP-seq data. Moreover, they also suggest that studying the epigenetic regulation of the GABAergic signaling pathway may contribute to in-depth understanding of these excitotoxicity effects.

In conclusion, we demonstrated that the EZH2 inhibitor GSK-126 alleviates neuronal apoptosis in ischemic stroke by inhibiting H3K27me3. Furthermore, activation of the 
ERK-MAPK pathway is required for the neuroprotective function of GSK-126 in the ischemic brain.

Supplementary Information The online version contains supplementary material available at https://doi.org/10.1007/s12035-021-02677-3.

Acknowledgements We thank Prof. Guiying Yao of Xinxiang Medical University for her assistance in animal modeling. We thank Cloudseq Biotech Inc (Shanghai, China) for high-throughput sequencing service and bioinformatic support.

Author Contribution ZW and SQ conceived this experiment. YS, LZ, and TL performed the experiments. The manuscript was drafted by $\mathrm{ZW}$ and YS. LL and LZ contributed to editing the manuscript. All authors read and approved the final version of the manuscript.

Funding This work was supported by the National Natural Sciences Foundation of China (grant numbers 81801168, 81802063).

Data Availability The data that support the findings of this study are available from the corresponding author upon reasonable request.

\section{Declarations}

Ethical Approval All animal procedures were approved by the committee on the ethics of animal experiments of Xuzhou Medical University, and animals were cared for in accordance with the National Institute of Health Guide for the Care and Use of Laboratory Animals.

Consent to Participate Not applicable.

Consent for Publication Not applicable.

Conflict of Interest The authors declare no competing interests.

Open Access This article is licensed under a Creative Commons Attribution 4.0 International License, which permits use, sharing, adaptation, distribution and reproduction in any medium or format, as long as you give appropriate credit to the original author(s) and the source, provide a link to the Creative Commons licence, and indicate if changes were made. The images or other third party material in this article are included in the article's Creative Commons licence, unless indicated otherwise in a credit line to the material. If material is not included in the article's Creative Commons licence and your intended use is not permitted by statutory regulation or exceeds the permitted use, you will need to obtain permission directly from the copyright holder. To view a copy of this licence, visit http://creativecommons.org/licenses/by/4.0/.

\section{References}

1. Barthels D, Das H (2020) Current advances in ischemic stroke research and therapies. Biochim Biophys Acta Mol Basis Dis 1866(4): 165260

2. Cheng CY, Kao ST, Lee YC (2020) Angelica sinensis extract protects against ischemia-reperfusion injury in the hippocampus by activating p38 MAPK-mediated p90RSK/p-Bad and p90RSK/ CREB/BDNF signaling after transient global cerebral ischemia in rats. J Ethnopharmacol 252:112612
3. Liu PP et al (2018) Polycomb repressive complex 2: emerging roles in the central nervous system. Neuroscientist 24(3):208-220

4. Li J et al (2013) EZH2-mediated H3K27 trimethylation mediates neurodegeneration in ataxia-telangiectasia. Nat Neurosci 16(12):1745-1753

5. Wang $Z$ et al (2017) Decreased methylation level of H3K27me3 increases seizure susceptibility. Mol Neurobiol 54(9):7343-7352

6. Xiao J et al (2020) Long-term probiotic intervention mitigates memory dysfunction through a novel H3K27me3-based mechanism in lead-exposed rats. Transl Psychiatry 10(1):25

7. $\mathrm{Ma} \mathrm{Z}$ et al (2018) Epigenetic drift of H3K27me3 in aging links glycolysis to healthy longevity in Drosophila. Elife 7:e35368

8. Roy-O'Reilly M, McCullough LD (2018) Age and sex are critical factors in ischemic stroke pathology. Endocrinology 159(8):3120-3131

9. Yu YL et al (2013) Smurf2-mediated degradation of EZH2 enhances neuron differentiation and improves functional recovery after ischaemic stroke. EMBO Mol Med 5(4):531-547

10. Chen J et al (2019) EZH2 inhibitor DZNep modulates microglial activation and protects against ischaemic brain injury after experimental stroke. Eur J Pharmacol 857:172452

11. Wang $Z$ et al (2021) The role of EZH2 inhibitor, GSK-126, in seizure susceptibility. J Mol Neurosci 71(3):556-564

12. Pulsinelli WA, Brierley JB (1979) A new model of bilateral hemispheric ischemia in the unanesthetized rat. Stroke 10(3):267-272

13. Hao L et al (2016) Neuroprotective effects of inhibiting Fyn $\mathrm{S}$-nitrosylation on cerebral ischemia/reperfusion-induced damage to CA1 hippocampal neurons. Int J Mol Sci 17(7):1100

14. Chen J et al (2001) Intravenous administration of human umbilical cord blood reduces behavioral deficits after stroke in rats. Stroke 32(11):2682-2688

15. Jiang M et al (2014) Neuroprotective effects of bilobalide on cerebral ischemia and reperfusion injury are associated with inhibition of pro-inflammatory mediator production and down-regulation of JNK1/2 and p38 MAPK activation. J Neuroinflammation 11:167

16. Mehta SL, Manhas N, Raghubir R (2007) Molecular targets in cerebral ischemia for developing novel therapeutics. Brain Res Rev 54(1):34-66

17. Duncia JV et al (1998) MEK inhibitors: the chemistry and biological activity of U0126, its analogs, and cyclization products. Bioorg Med Chem Lett 8(20):2839-2844

18. Guo X et al (2019) Calcitriol alleviates global cerebral ischemiainduced cognitive impairment by reducing apoptosis regulated by VDR/ERK signaling pathway in rat hippocampus. Brain Res 1724:146430

19. Yu Z et al (2018) Neuroprotective effects of Tongxinluo on focal cerebral ischemia and reperfusion injury in rats associated with the activation of the MEK1/2/ERK1/2/p90RSK signaling pathway. Brain Res 1685:9-18

20. Datta A et al (2020) Cell death pathways in ischemic stroke and targeted pharmacotherapy. Transl Stroke Res 11(6):1185-1202

21. Jhelum $P$ et al (2017) Implications of epigenetic mechanisms and their targets in cerebral ischemia models. Curr Neuropharmacol 15(6):815-830

22. Palomer E et al (2016) Neuronal activity controls Bdnf expression via polycomb de-repression and CREB/CBP/JMJD3 activation in mature neurons. Nat Commun 7:11081

23. Zhang $\mathrm{H}$ et al (2018) Inhibiting Jumoji domain containing protein 3 (JMJD3) prevent neuronal apoptosis from stroke. Exp Neurol 308:132-142

24. Molinaro P et al (2008) Targeted disruption of $\mathrm{Na}+/ \mathrm{Ca} 2+$ exchanger 3 (NCX3) gene leads to a worsening of ischemic brain damage. J Neurosci 28(5):1179-1184

25. McCabe MT et al (2012) EZH2 inhibition as a therapeutic strategy for lymphoma with EZH2-activating mutations. Nature 492(7427):108-112 
26. Fioravanti R et al (2018) Six years (2012-2018) of researches on catalytic EZH2 inhibitors: the boom of the 2-pyridone compounds. Chem Rec 18(12):1818-1832

27. Yap TA et al (2019) Phase I study of the novel enhancer of zeste homolog 2 (EZH2) inhibitor GSK2816126 in patients with advanced hematologic and solid tumors. Clin Cancer Res 25(24):7331-7339

28. Zhang $\mathrm{P}$ et al (2015) ABCB1 and ABCG2 restrict the brain penetration of a panel of novel EZH2-Inhibitors. Int J Cancer 137(8):2007-2018

29. Liang J et al (2015) Nicotinamide mononucleotide adenylyltransferase 1 protects neural cells against ischemic injury in primary cultured neuronal cells and mouse brain with ischemic stroke through AMP-activated protein kinase activation. Neurochem Res 40(6):1102-1110

30. Uzdensky AB (2019) Apoptosis regulation in the penumbra after ischemic stroke: expression of pro- and antiapoptotic proteins. Apoptosis 24(9-10):687-702

31. Dou B et al (2018) Buyang huanwu decoction attenuates infiltration of natural killer cells and protects against ischemic brain injury. Cell Physiol Biochem 50(4):1286-1300

32. Zeng $\mathbf{J}$ et al (2019) Metformin protects against oxidative stress injury induced by ischemia/reperfusion via regulation of the lncRNA-H19/miR-148a-3p/Rock2 axis. Oxid Med Cell Longev 2019:8768327

33. Moskowitz MA, Lo EH, Iadecola C (2010) The science of stroke: mechanisms in search of treatments. Neuron 67(2):181-198

34. Singh V et al (2019) Modes of calcium regulation in ischemic neuron. Indian J Clin Biochem 34(3):246-253

35. Lai TW, Zhang S, Wang YT (2014) Excitotoxicity and stroke: identifying novel targets for neuroprotection. Prog Neurobiol 115:157-188

36. Li H et al (2013) Inhibition of the group I mGluRs reduces acute brain damage and improves long-term histological outcomes after photothrombosis-induced ischaemia. ASN Neuro 5(3):195-207

37. Xie W et al (2018) Protective effects and target network analysis of ginsenoside $\mathrm{Rg} 1$ in cerebral ischemia and reperfusion injury: a comprehensive overview of experimental studies. Cells 7(12):270
38. Zhen $\mathrm{Y}$ et al (2016) Activation of the calcium-sensing receptor promotes apoptosis by modulating the JNK/p38 MAPK pathway in focal cerebral ischemia-reperfusion in mice. Am J Transl Res 8(2):911-921

39. Lee $\mathrm{SH}$ et al (2010) ERK activation drives intestinal tumorigenesis in Apc(min/+) mice. Nat Med 16(6):665-670

40. Shaul YD, Seger R (2007) The MEK/ERK cascade: from signaling specificity to diverse functions. Biochim Biophys Acta 1773(8):1213-1226

41. Namura $S$ et al (2001) Intravenous administration of MEK inhibitor U0126 affords brain protection against forebrain ischemia and focal cerebral ischemia. Proc Natl Acad Sci USA 98(20):11569-11574

42. Li J et al (2018) U0126 protects hippocampal CA1 neurons against forebrain ischemia-induced apoptosis via the ERK1/2 signaling pathway and NMDA receptors. Neurol Res 40(4):318-323

43. Lie MEK et al (2017) Glial GABA transporters as modulators of inhibitory signalling in epilepsy and stroke. Adv Neurobiol 16:137-167

44. Pamenter ME et al (2012) Painted turtle cortex is resistant to an in vitro mimic of the ischemic mammalian penumbra. J Cereb Blood Flow Metab 32(11):2033-2043

45. Ouyang C et al (2007) Enhanced activity of GABA receptors inhibits glutamate release induced by focal cerebral ischemia in rat striatum. Neurosci Lett 420(2):174-178

46. Bhattacharya $P$ et al (2014) Alleviation of glutamate mediated neuronal insult by piroxicam in rodent model of focal cerebral ischemia: a possible mechanism of GABA agonism. J Physiol Biochem 70(4):901-913

Publisher's Note Springer Nature remains neutral with regard to jurisdictional claims in published maps and institutional affiliations. 